\title{
Turbulent magnetic energy spectrum and the cancellation function of solar photospheric magnetic fields
}

\author{
G. Marschalkó ${ }^{1,2, \star}$ and K. Petrovay ${ }^{1}$ \\ 1 Eötvös University, Department of Astronomy, Budapest, P.O. Box 32, H-1518, Hungary \\ ${ }^{2}$ Konkoly Observatory of the Hungarian Academy of Sciences
}

The dates of receipt and acceptance should be inserted later

Key words Sun: atmosphere - magnetic fields - turbulence

\begin{abstract}
A simple analytical relation of form $\alpha=2 \kappa-1$ between the magnetic energy spectral exponent $\alpha$ of the turbulent magnetic field in the solar photosphere and its magnetic flux cancellation exponent $\kappa$, valid under certain restrictive assumptions, is tested and extended outside its range of validity in a series of Monte Carlo simulations. In these numerical tests artificial "magnetograms" are constructed in 1D and 2D by superposing a discrete set of Fourier modes of the magnetic field distribution with amplitudes following a power law spectrum and measuring the cancellation function on these simulated magnetograms. Our results confirm the validity of the analytical relation and extend it to the domain $\alpha<-1$ where $\kappa \rightarrow 0$ as $\alpha \rightarrow-\infty$. The observationally derived upper limit of 0.38 on $\kappa$ implies $\alpha<-0.24$ in the granular size range, apparently at odds with a small scale dynamo driven in the inertial range.
\end{abstract}

(c) 2013 WILEY-VCH Verlag GmbH \& Co. KGaA, Weinheim

\section{Introduction}

The structure of the magnetic field in the solar photosphere is still far from being fully understood (for reviews see de Wijn et al. 2007, Martínez Pillet 2012). The two main methods of studying solar magnetism are based on the Zeeman and Hanle effects, respectively. As the above mentioned effects display different sensitivity to various field configurations depending on their amplitude and spatial organization, we have a dichotomic view of the magnetism of the solar photosphere.

The basic properties of the magnetic network, consisting of kilogauss strength flux tubes, have been clarified by longitudinal Zeeman magnetometry decades ago. The contribution of these elements to the large scale unsigned flux density $\langle|B|\rangle$ typically does not exceed a few Gauss in quiet sun regions. Yet the analysis of the Hanle effect depolarization of spectral lines indicates that the total value of $\langle|B|\rangle$ is well in excess of $100 \mathrm{G}$ (Trujillo Bueno et al. 2004). Most of this flux was, then, previously hidden to traditional Zeeman magnetometry, presumably due to its fine scale turbulent structuring that leads to the cancellation of the net Zeeman polarization signal inside a resolution element.

It has long been suspected that the sporadic internetwork (IN) magnetic flux concentrations seen in longitudinal magnetograms represent the observable part of this hidden or turbulent magnetic field. Recent improvement in the resolution and sensitivity of polarimeters, in particular the SDO/HMI (Solar Dynamics Observatory/Helioseismic and Magnetic Imager), Hinode/SP (Spectropolarimeter) and

^ Corresponding author: e-mail: G.Marschalko@ astro.elte.hu
Sunrise/IMaX (Imaging Magnetograph eXperiment) instruments, have led to a breakthrough in the $3 \mathrm{D}$ vector polarimetry of the photospheric magnetic field, clearly demonstrating the presence of a large number of IN flux concentrations. In contrast to the nearly vertical, $\mathrm{kG}$ network flux tubes, these IN concentrations only reach hectogauss field strengths and their orientation may be either more or less horizontal or vertical. Statistically, the distribution of magnetic field orientations in this turbulent field seems to be more or less isotropic, perhaps with some (currently debated) preference towards the horizontal direction.

Given that this observed IN field now represents a nonnegligible fraction of the formerly "hidden" turbulent flux, its detection offers a unique chance to empirically study MHD turbulence in a compressible, stratified plasma. In this respect, recent studies of the cancellation function, $\chi\left(l_{0}\right)$, of the photospheric magnetic field are of interest. The function $\chi\left(l_{0}\right)$ is defined as the unsigned flux density $\langle|B|\rangle$ seen at a finite resolution $l_{0}$ in a longitudinal magnetogram, normalized to the intrinsic total unsigned flux density $\langle|B|\rangle_{0}$ :

$$
\chi\left(l_{0}\right)=\langle|B|\rangle\left(l_{0}\right) /\langle|B|\rangle_{0} .
$$

Analyzing a Hinode/SP magnetogram, Pietarila Graham, Danilovic and Schüssler (2009) found that the shape of the cancellation function is a power law $\chi\left(l_{0}\right) \propto l_{0}^{-\kappa}$ in the range 0.2 to $20 \mathrm{Mm}$, with $\kappa=0.26$. The analysis was repeated by Stenflo (2011) upon recalibrating the magnetogram, yielding a value $\kappa=0.127$, independently confirmed also by the analysis of Hinode/NFI magnetograms. Recently, Pietarila and Pietarila Graham (2012) have made an extensive comparative analysis of the cancellation functions resulting from SoHO/MDI, SDO/HMI and Hinode/SP magnetograms, finding that the derived $\kappa$ values are heavily 


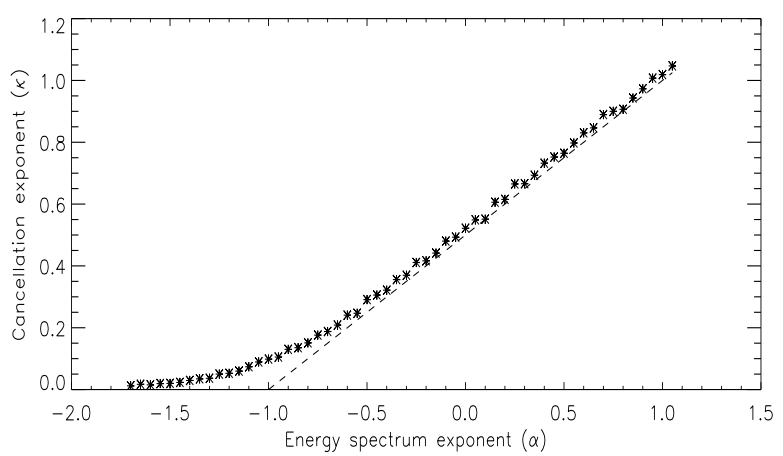

Fig. 1 Cancellation exponent $\kappa$ as a function of the magnetic energy spectral exponent $\alpha$ in the 1D case, with the analitically derived relation (dashed line).

influenced by instrument noise, seeing effects, net flux imbalance in the field and by the proper exclusion of field components not belonging to the turbulent field (such as netwok elements). They find that the $\kappa$ values derived for quiet regions decrease with improving instrument quality, i.e. along the MDI $\rightarrow$ HMI $\rightarrow$ SP series, so that the SP value of 0.38 can be considered a conservative upper bound for the true value of the cancellation exponent. Lower values reported in earlier studies were apparently due to improper masking of network fields and/or the use of fields of view with a higher flux imbalance (i.e. less typical quiet sun areas).

As the most important theoretical tool in the study of the scaling behaviour of turbulent flows is the energy spectral function, the question naturally arises how $\chi\left(l_{0}\right)$ is related to the magnetic energy spectral function, written as $E_{k} \sim k^{\alpha}$ ?

In Section 2 we derive a simple analytical relation between the cancellation exponent, $\kappa$, and the spectral exponent, $\alpha$, valid under some assumptions. The relation is extended beyond the limit of validity of the analytic formula in a set of Monte Carlo simulations in Section 3. Conclusions are drawn in Section 4.

\section{Analytical treatment of the problem}

Consider a fluctuating 2D field of zero mean, such as the residual magnetic field upon subtracting the large scale mean field from a magnetogram. If the actual longitudinal residual magnetic field distribution is denoted by $B_{0}(x, y)$ then the residual magnetogram observed at a finite resolution $l_{0}$ is

$$
B(x, y)=\iint B_{0}\left(x^{\prime}, y^{\prime}\right) \operatorname{PSF}\left(x-x^{\prime}, y-y^{\prime}\right) d x^{\prime} d y^{\prime}
$$

where PSF is the point spread function for the given resolution $l_{0}$, and $\mathrm{x}, \mathrm{y}$ are Cartesian coordinates of the magnetogram. The Fourier transform of equation (2) is

$$
\hat{B}\left(k_{x}, k_{y}\right)=\hat{B}_{0}\left(k_{x}, k_{y}\right) \cdot \operatorname{MTF}\left(k_{x}, k_{y} ; k_{0}\right)
$$

where the modulation transfer function MTF is the Fourier transform of the PSF, $k_{x}^{2}+k_{y}^{2}=k^{2}$ and $k_{0}=\pi / l_{0}$. For statistical isotropy in the plane the MTF only depends on the modulus of the wave number $(k)$. For simplicity we also assume that there is only one characteristic resolution scale involved: in this case the MTF will only depend on the combination $k / k_{0}$.

Let the spectrum of the magnetic energy (in the line of sight component) as a function of wave number be $E_{k}$. From elementary turbulence theory we have

$$
E_{k}=\frac{1}{2} \hat{B}(k) \hat{B}^{*}(k)
$$

(As with $B, E_{k}$ is the spectrum observed at finite resolution while $E_{0, k}$ is the actual spectrum and $\hat{B}^{*}(k)$ is the complex conjugate of $\hat{B}(k)$.)

Assuming a power law turbulent magnetic energy spectrum

$$
E_{0, k}=E_{0, k_{0}}\left(\frac{k}{k_{0}}\right)^{\alpha}
$$

with $\alpha>-1$, the total magnetic energy observed at resolution $l_{0}$ can be calculated by means of the MTF in the following way:

$$
\begin{array}{r}
E\left(k_{0}\right)=\int_{0}^{\infty} E_{k} d k=\int_{0}^{\infty} E_{0, k}\left|\operatorname{MTF}\left(k / k_{0}\right)\right|^{2} d k \\
=k_{0} E_{0, k_{0}} \int_{0}^{\infty} \tilde{k}^{\alpha}|\operatorname{MTF}(\tilde{k})|^{2} d \tilde{k}
\end{array}
$$

where we used the substitution $\tilde{k}=k / k_{0}$.

The derivative of equation (6) by $k_{0}$ is:

$$
E^{\prime}\left(k_{0}\right)=E_{0, k_{0}} \int_{0}^{\infty} \tilde{k}^{\alpha}|\operatorname{MTF}(\tilde{k})|^{2} d \tilde{k}
$$

This demonstrates that, independently of the form of the MTF, the rate of increase in magnetic energy seen with increasing resolution is proportionate to the value $E_{0, k_{0}}$ of the magnetic energy spectral function at the inverse resolution, $k_{0}$.

To find a relation between this rate of increase and the cancellation function we need to make a further assumption regarding the self-similarity (fractal nature) of the magnetic field distribution $B_{0}(x, y)$. Let us introduce the notation $F=\left\langle B^{2}\right\rangle /\langle|B|\rangle^{2}$. The probability density function (PDF) of $|B|$ will determine the value of $F$, but for a scale invariant (fractal) field distribution $F$ should be resolution independent. This suggests that $\left\langle B^{2}\right\rangle \sim k_{0}^{2 \kappa}$ if $\langle|B|\rangle \sim k_{0}^{\kappa}$. The derivative of this yields by Eq. (7) $E_{0, k} \sim k^{2 \kappa-1}$ i. e.

$$
\alpha=2 \kappa-1 .
$$

Note that this formula is the non-intermittent special case of the more general relationship derived by Vainshtein et al. (1994). While the derivation given above is more restricted in its assumptions regarding intermittency, it does include the effects of finite instrumental resolution and its transparency makes it easy to see the limitations of validity 


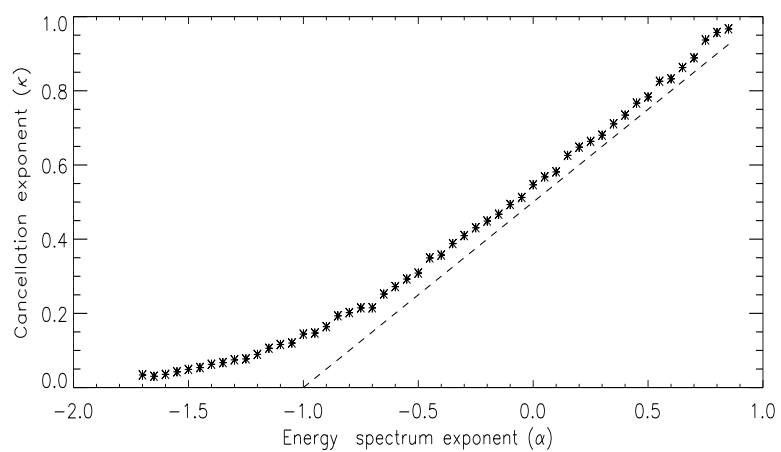

Fig. 2 Cancellation exponent $\kappa$ as a function of the magnetic energy spectral exponent $\alpha$ in the 2D case, with the analitically derived relation (dashed line).

of equation (8). Indeed, it should be stressed that this relationship is valid only for $\alpha>-1$. For $\alpha<-1$ equation (8) would result in negative values for $\kappa$ which is clearly impossible in view of the meaning of the cancellation function. Indeed, for $\alpha<-1$ the integral on the r.h.s. of equation (7) would diverge at its lower limit (as $|M T F|(k \rightarrow 0) \rightarrow 1$ ). In this case it needs to be taken into account that the power law range in the spectrum can only extend down to an integral scale $k_{1}$, replacing zero as the lower bound of the integral, and the value of the integral will be dominated by the contribution near $k_{1}$, asymptotically making $E\left(k_{0}\right)$ and $\chi$ independent of $k_{0}$. Our expectation, then, is that $\kappa \rightarrow 0$ as $\alpha \rightarrow-\infty$. Due to the dependence on a preferred scale $1 / k_{1}$ the cancellation function is expected to deviate from power law behaviour in this regime.

We further note that, depending on the form of the MTF and the value of $\alpha$, the integral in equation (7) may also diverge at its upper limit. This may set an upper limit on $\alpha$ for the validity of our analytical formula (and of the power law form of $\chi$ ). As, however, this depends on the particular form of the MTF used, this upper limit is less generic than the lower bound $\alpha>-1$ discussed above.

\section{Monte Carlo simulations}

To demonstrate the validity of the analytical relation (8) as well as to extend the $\kappa-\alpha$ relationship into the range $\alpha \lesssim-1$ we set up a series of Monte Carlo simulations. First we do this for the 1D case, for conceptual simplicity and numerical advantages.

We represent the magnetic field by a discrete set of $n$ modes, equidistant with spacing 1 on a base- 2 logarithmic scale. Assuming power law energy spectra with different exponents, the energy between $k_{0}$ and $2 k_{0}$ is

$$
\int_{k_{0}}^{2 k_{0}} E_{k} d k=\int_{\log _{2}\left(k_{0}\right)}^{\log _{2}\left(k_{0}\right)+1} k E_{k} d \log _{2} k
$$

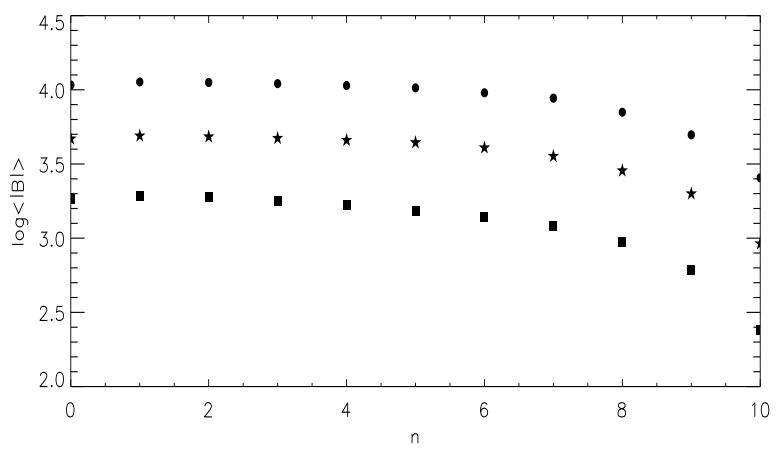

Fig. 3 Cancellation function with different turbulent magnetic energy spectra. Dots: $\alpha=-1.7$; stars: $\alpha=-1.6$; squares: $\alpha=-1.5$.

Since $k E_{k} \sim k^{1+\alpha}$ we have $B_{k} \sim k^{(1+\alpha) / 2}$, so the Fourier amplitude of each mode is:

$$
\hat{B}_{j}=\hat{B}_{j, 0} e^{i \phi_{j}} \text { where } \hat{B}_{j, 0} \sim k^{(1+\alpha) / 2}
$$

where $j=1 . . n$ and the phases $\phi_{j}$ were assumed to be random. (We use $n=14$ in the $1 \mathrm{D}$ case.) The computation was performed on a grids of $N=2^{n}$ points, the modes considered having $k_{j}=2 \pi / 2^{j}(j=1 . . n) . B$ reads

$$
B=\sum_{j=1}^{n} \hat{B}_{j} e^{i k_{j} x}+\sum_{j=1}^{n} \hat{B}_{j}^{*} e^{-i k_{j} x}
$$

Having generated the function B on the full grid, we smear it with various "masks" or grids of coarser resolution ( $2^{j}$ grid points with $j=1 . . n$ ), at different random positions, and we calculate the resulting flux density $\langle|B|\rangle$. The $\alpha$ values are taken from the range [-3,3], with a denser sampling in the interval $[-0.9,0]$. For each value of $\alpha$, the computation is done for a large number of random phase sets $\left\{\phi_{j}\right\}$ and mask positions, averaging the results. The exponent of the cancellation function was determined by a power law fit to all points except the two largest scales.

In the next step the calculation is performed again for the $2 \mathrm{D}$ case with the same method. Due to the higher computational requirements the maximum grid size, and hence the number of modes was lower $(n=12)$ than in the 1D case.

The resulting $\alpha-\kappa$ relation is plotted in Figures 1 and 2 for the 1D and 2D case, respectively. It is apparent that within its range of validity the analytical relation (8) indeed provides an excellent description of the relationship, down to $\alpha$ values of -0.7 or so. Towards large negative values of the spectral exponent we have $\kappa \rightarrow 0$ as expected.

Figures 3 and 4 present the cancellation functions obtained with different $\alpha$ values. It is apparent that for values within the scope of the analytical relation (Figure 4) the cancellation function can be fairly well estimated by a power law. On the other hand, for lower $\alpha<-1$ (Figure 3) the cancellation function shows a significant deviation from 
power law behaviour, as expected (cf. the discussion at the end of Section 2.)

The conservative upper limit of $\kappa<0.38$ obtained by Pietarila and Pietarila Graham (2012) implies $\alpha<-0.24$ according to our analytical relation (8), confirmed by the numerical results. The much more stringent result obtained by Stenflo (2011), on the other hand, would imply $\alpha \simeq-0.95$ based on our numerical results. (The analytical relation would give -0.746 ).

\section{Conclusion}

Under the assumption of a self-similar (fractal) magnetic field structure we have derived a simple analytical relationship, equation (8), between $\kappa$ and $\alpha$. The relation is expected to be valid for $\alpha>-1$. The relation is tested and extended outside its range of validity in a series of Monte Carlo simulations. In these numerical tests artificial "magnetograms" are constructed in 1D and 2D by superposing a discrete set of Fourier modes of the magnetic field distribution with amplitudes following a power law spectrum and measuring the cancellation function on these simulated magnetograms. Our results confirm the validity of the analytical relation and extend it to the domain $\alpha<-1$ where $\kappa \rightarrow 0$ with decreasing $\alpha$ values.

The observationally derived upper limit of 0.38 on $\kappa$ implies $\alpha<-0.24$. The lowest $\kappa$ values detected in any magnetogram field to date would correspond to $\alpha \simeq-0.95$. These findings provide evidence that the magnetic energy spectral function is a decreasing function of wavenumber in the granular size range $(0.2$ to $20 \mathrm{Mm})$, in contrast to the prediction of small scale dynamo simulations where $\alpha>0$ is found (Schekochihin et al. 2007, Pietarila Graham et al. 2010). The limits we derived do not exclude the possibility of an $\alpha=-1$ spectrum which is the hallmark of a larger scale field being passively advected by turbulent motions. This might indicate that the photospheric turbulent magnetic field results from passive field amplification; however, in this case we would expect $\langle|B|\rangle \sim|\langle B\rangle|$ which is not observed (Lites 2011). This suggests that the turbulent magnetic field in the solar photosphere does originate in a dynamo but this dynamo does not operate in the inertial range as in current simulations but rather at or above the integral scale. This may be due to the fact the magnetic Prandtl number $P_{m}$ in the solar plasma is much lower than in current numerical simulations. There are indications that at such low values of, $P_{m}$, the critical magnetic Reynolds number, $R_{m}$, for dynamo action is much higher than for $P_{m} \simeq 1$, which may impede the operation of an inertial range dynamo. At larger scales, however, $R_{m}$ may be high enough to drive a dynamo; or alternatively a small scale dynamo driven by a fundamentally different process might be at work at or above the integral scale.

Our constraints on $\alpha$ are in accordance with the value of $\alpha \simeq-1.3$ derived by Abramenko et al. (2001) for quiet sun regions from a direct analysis of magnetograms.

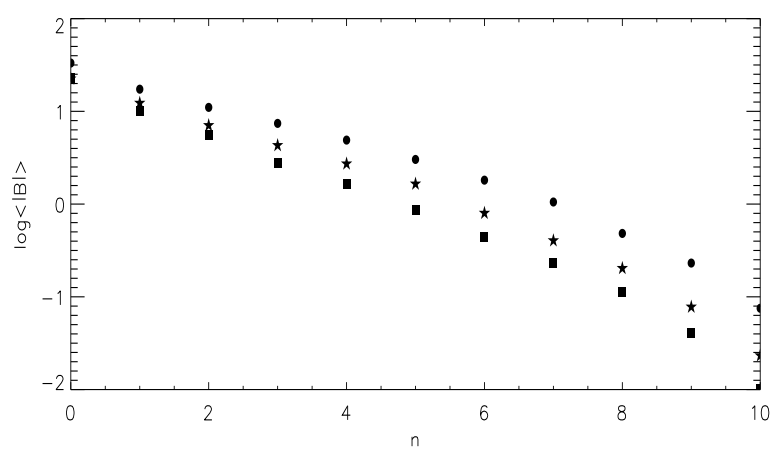

Fig. 4 Cancellation function with different turbulent magnetic energy spectra. Dots: $\alpha=-0.7$; stars: $\alpha=-0.6$; squares: $\alpha=-0.5$.

The single most important remaining restriction in our numerical results is the assumption of random phases (i.e. lack of intermittency). The photospheric turbulent magnetic field is known to be distributed in a very distinctive pattern, forming IN field concentrations, following intergranular lanes and mesogranular structure. This is very different from the amorphous superposition of Fourier modes with random phases. Whether, and to what extent non-random phases might influence our inferences should be the subject of future research.

Acknowledgements. This research was supported by the Hungarian Science Research Fund (OTKA) grants no. K81421 and K83133. This project has been also supported by ESTEC Contract No. 4000106398/12/NL/KML.

\section{References}

Abramenko, V.; Yurchyshyn, V.; Wang, H.; Goode, P. R.: 2001, SoPh 201, 225

de Wijn, A. G.; Stenflo, J. O.; Solanki, S. K.; Tsuneta, S.: 2009, Space Sci. Rev. 144, 275

Lites, B. W.: 2011, ApJ 737, 52

Martínez Pillet, V.: 2013, arXiv:1301.6933

Pietarila Graham, J., Danilovic, S., Schüssler, M.: 2009, ApJ 693, 1728

Pietarila Graham, J., Cameron, R., Schüssler, M.: 2010, ApJ 714, 1606

Pietarila, A., Pietarila Graham, J.: 2012, SoPh, Online First

Schekochihin, A. A.; Iskakov, A. B.; Cowley, S. C.; McWilliams, J. C.; Proctor, M. R. E.; Yousef, T. A.: 2007, New J. Phys. 9, 300

Stenflo, J. O.: 2011, A\&A 529, A42

Trujillo Bueno, J., Shchukina, N., Asensio Ramos, A. 2004, Nature, 430, 326

Vainshtein S. I., Du, Y., Sreenivasan, K. R. 1994, Phys. Rev. E, 49, $\mathrm{R} 2521$ 